\title{
Battery Power Management Routing Considering Participation Duration for Mobile Ad Hoc Networks
}

\author{
Masaru Yoshimachi and Yoshifumi Manabe
}

\begin{abstract}
We propose a new routing protocol BPMR (Battery Power Management Routing) for MANET (Mobile Ad Hoc NETwork). This protocol maintains high availability of network nodes. The duration time of participating to a MANET differs among nodes. Each node must work as a relay node during the participation and consume its battery. It is not fair that the nodes participating to the MANET for a long time relay many packets and the ones participating for a short period relay few packets. MANET protocols should reduce the relay burden to the nodes that have contributed to the MANET for a long time. MBCR (Minimum Battery Cost Routing) was proposed for MANET, in which the route selection considers each node's remaining battery in order to improve the availability of MANET. In order to improve the availability, the nodes must not fall out early because of the battery exhaust. We propose BPMR which considers both of the participation duration and the remaining battery for the path selection. BPMR achieves fairness among nodes and high availability. The total remaining battery capacity by BPMR is superior to the one by MBCR. We show the effectiveness of BPMR by network simulations.
\end{abstract}

Index Terms-Battery consumption, fairness, MANET, MBCR, participation duration, routing protocol.

\section{INTRODUCTION}

Because of recent advances of the wireless network technology, there are wireless devices everywhere in our daily life. Most networks are based on an infrastructure that uses access points as wireless communication base stations. MANET (Mobile Ad Hoc NETwork) [1] does not require such wireless communication base stations. It is expected as a new type of wireless network. The paper [1] insists that MANET will see widespread use within the next few years. MANET is an independent network in which devices communicate only by their equipped wireless communication modules. MANET is expected to be used as a temporary network for mobile phones when the cellular network infrastructure is not available in the case of a disaster. It is also expected to be used in the mountain areas where there are no fixed-line networks. Devices within the scope of their radio waves can directly communicate with each other. In addition, each device has a function as a router. Thus a device can communicate to a device outside the scope of its radio wave, because the devices can relay the other devices' packets. Wireless devices often move in MANET. Thus the network topology changes with time. In MANET, the route to a destination device changes frequently because of the

Manuscript received September 30, 2015; revised January 20, 2016. This work was supported by JSPS KAKENHI Grant Number 26330019.

The authors are with Kogakuin University, Tokyo, 163-8677 Japan (e-mail: em15021@ns.kogakuin.ac.jp, manabe@cc.kogakuin.ac.jp). movement of the devices. Thus the routing protocols for MANET need to reconstruct the routing paths soon when the network topology changes.

Another usage of MANET is avoiding wireless network congestion. The traffic of cellular networks rapidly increases by mobile phones, thus the communication speed decreases. To avoid the decrease, load balancing is necessary. Currently, Wi-Fi spots are operated near many train stations for the purpose. However, setting up Wi-Fi spots is not easy and Wi-Fi has a disadvantage of its narrow effective radio range. Thus, there are areas where the load balancing cannot be easily performed. In order to solve the problem, short range communication among the users within the area can be performed by MANET, thus only long distance communication uses the infrastructure and load valancing is achieved. These types of works [2], [3] aim to improve the throughput. The paper [2] tried to reduce collisions for throughput improvement (MAC layer) in MANET. The authors showed the improvement by a simulation. The paper [3] compared famous protocols (DSDV, DSR and AODV) from the point of the number of packets, throughput, and delay by a simulation. Authors insisted that the best protocol is DSR.

Some protocols for MANET have been proposed to achieve low power consumption [4]-[6], however very few works have been done for the following fair load valancing problem.

In current MANET protocols, every device has the same probability to be selected as a relay device at the path selection. It is not fair that the devices participating to the MANET for a long time relays many packets and the ones participating for a short period relay few packets. Since devices wish for high speed communication and the minimum delay, high performance devices tend to be selected as the relay devices. Thus, these devices use more battery power than the other devices. Since the battery capacity of devices is limited, heavy load devices fall out from the network within a short period. It results in decreasing the success rate of communication and throughput of the network. Thus, we need a new protocol that aims long term communication stability. To achieve the purpose, the route selection must consider power consumption.

There are several protocols [7], [8] for MANET that considers the power consumption. MBCR [7] avoids to use low remaining battery devices, thus such devices tend not to fall out from the network early. However, MBCR sometimes increases the total power consumption in order to keep devices alive. It is important to keep devices alive in MANET but increasing the total power consumption is not desirable. DSR [8] was designed as a reactive type routing protocol for MANET. This protocol is one of the most protocols for 
MANET and registered as RFC 4728. DSR is simple and efficient. However it has some problems in the security, power consumption, fairness, and so on because of its simpleness. The paper [4] indicated that the one of the most important issue of routing protocols is to provide energy efficient routes and the transmission power control is necessary. The optimal adjustment of the power level is essential not only for energy conservation but also for the interference control. The paper [6] insisted that adjusting the transmit powers of nodes in a multi hop path to the same level is important [4]. The paper [5] indicated that the power conservation is a critical issue for MANET. Some spanning tree protocols were compared by simulations.

We propose a new protocol, BPMR (Battery Power Management Protocol), that achieves the viability and maximizing the number of alive devices. BPMR has the both of the characteristics of avoiding low battery devices by MBCR and avoiding long participation duration devices. The route selection by BPMR avoids including low battery devices in the route. In addition, BPMR considers participation duration time in MANET. Thus the devices can prolong their battery life and the number of available nodes is kept high in the network. BPMR selects the most suitable path as a route from a source device to a destination node among the paths between the source node and the destination node. There are proactive type protocols, reactive type protocols, and hybrid type protocols for MANET routing protocols. Though we consider reactive type routing protocols in this paper, the idea of BPMR can be applied to the other types of routing protocols. We compare BPMR with the other protocols by simulations. BPMR keeps the availability of devices high for a longer time than the other routing protocols.

The rest of this paper is organized as follows. Section II shows current routing protocols for MANET. Section III shows problems in current routing protocols. In Section IV, we show the detail of the proposed protocol and the results of the simulations. We conclude the paper in Section V.

\section{CurRent Routing Protocols For MANET}

\section{A. DSR (Dynamic Source Routing)}

DSR [8] is a reactive type protocol that constructs communication paths on-demand. The routes are requested at the time when the communication is necessary. The route selection method is as follows. Note that in the rest of the paper, a wireless communication device is denoted by a node $x_{i}$. We show the detail of the path selection algorithm by DSR.

1) The source node generates a RREQ packet and the packet is broadcasted to the neighboring nodes in its effective radio wave range.

2) A node that receives the RREQ packet checks whether its destination node is itself. If it is not the destination, it writes its node ID to the RREQ packet and broadcasts the RREQ packet.

3) If a node that receives the RREQ packet is the destination node, it sends a RREP packet to the source node. A RREP packet is sent from the destination node to the node from which the RREQ packet arrived. The node that receives the RREP packet sends the RREP packet to the node from which the corresponding RREQ packet arrived. By repeating this procedure, a RREP packet is sent from the destination node to the source node through the reverse of the path that the RREQ packets are sent.

4) The source nodes select the route from which the first RREP packet arrived to the source node.

\section{B. MBCR (Minimum Battery Cost Routing)}

MBCR [7] adjusts the nodes' battery levels to improve the availability of MANET. MBCR is a routing path selection algorithm and can be used with any current routing protocols. At the path selection, MBCR selects the path with the maximum total remaining battery on the path. Node $x_{i}$ 's current remaining battery level (1-100) is denoted by BL $\left[x_{i}\right]$. The evaluation value of a path $P=\left(x_{1}, x_{2}, \ldots, x_{n}\right)$ from $x_{1}$ to $x_{n}$, PathValue $(P)$, is

$$
\operatorname{PathValue}(P)=\sum_{i=1}^{n} \frac{1}{B L\left[x_{i}\right]}
$$

where $x_{i}(2 \leq i \leq n-1)$ are the relay nodes. In the above equation, the evaluation value of a node is low if its battery level is high. For a set of paths obtained by RREPs, MBCR selects the following SelectPath as the route from $x_{1}$ to $x_{n}$.

$$
\text { SelectPath }=\operatorname{argmin}\{\operatorname{PathValue}(P) \mid P \in A\},
$$

where $A$ is the set of paths obtained by RREPs.

We show the detail of the path selection algorithm by MBCR. This algorithm inherits DSR thus we indicate additional and different parts from DSR algorithm.

1) The same as DSR.

2) A node that receives the RREQ packet checks whether its destination node is itself. If it is not the destination, it writes its node ID and its remaining battery level (BL) to the RREQ packet and broadcasts the RREQ packet.

3) The same as DSR.

4) The source node selects the route using (1) and (2) to the received RREP packets.

\section{Problems In CURRENT Routing Protocols}

DSR selects the minimum delay path at the path selection. It means that DSR tends to select the minimum hop path at the path selection. The most suitable node for relaying is always selected by DSR as a relay node. Thus, it is unfair that the amount of relay packets is different among nodes. Nodes might not participate in the MANET all the time. Some nodes might participate in MANET when they need to communicate with the other nodes and leave from MANET immediately after they finish their necessary communication. MANET can be established only when there are many volunteer participation nodes. Thus, quickly-leaving nodes are not beneficial for the entire MANET. On the other hand, some nodes might keep to participate to MANET for a long duration and forward many packets for the other nodes. Such nodes contribute very much to the entire MANET. In the point of view of fairness, the nodes that have contributed to 
the MANET must not be selected as a relay node. In current routing protocols, the possibility to be selected as a relay node is the same between a newly participated node and a node that has relayed many packets for the other nodes. We introduce this type of fairness to MANET routing protocols.

In addition, the number of participating nodes is important to obtain a dense network topology. When the battery is exhausted in a node, the node falls out from the MANET and the number of participating nodes decreases. It is not desirable for MANET. Therefore it is necessary to avoid falling out nodes by battery exhaustion. MBCR avoids falling out nodes and achieves high availability of MANET. In order to indicate this characteristic of MBCR, we executed a simulation to compare DSR and MBCR by self-made simulator using $\mathrm{C}$-language. The simulator locates 50 nodes in $500 \mathrm{~m} \times 500 \mathrm{~m}$ space and generates random communication requests. The moving speed of nodes is $4 \mathrm{~km} / \mathrm{h}$ in average. The nodes' maximum battery capacity is $5550 \mathrm{mWh}$ $(1500 \mathrm{mAh}, 3.7 \mathrm{~V})$. Each node's initial battery level is randomly selected between $1 \%$ and $100 \%$.

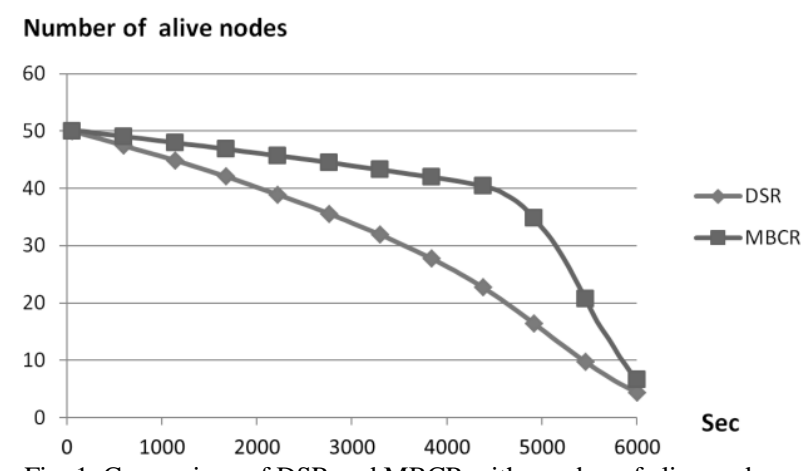

Fig. 1. Comparison of DSR and MBCR with number of alive nodes.

Fig. 1 shows the change of the number of alive nodes of DSR and MBCR by the simulation. In Fig. 1, MBCR decreases the number of falling out nodes more than DSR. Since MBCR considers the network availability, there are cases that the power consumption increases. MBCR does not select a path in which there is a very low battery level node and uses another path even if its number of hops is not the minimum. It results in increasing the total power consumption.

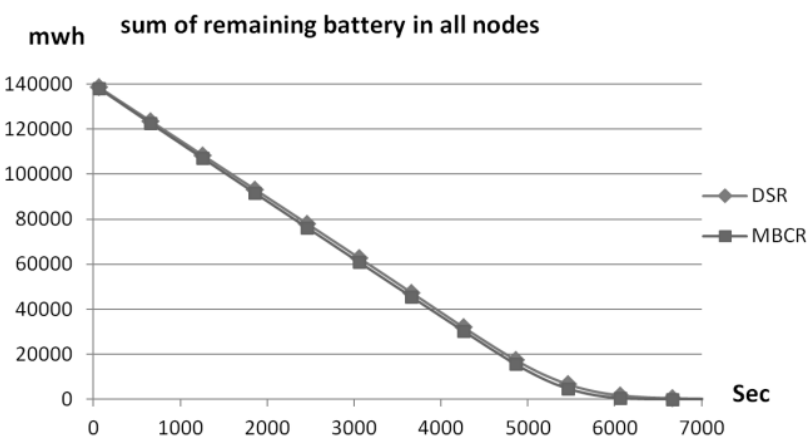

Fig. 2. Comparison of DSR and MBCR with sum of remaining battery capacity of all nodes.

Fig. 2 shows the change of the sum of remaining battery among alive nodes by the above simulation. In Fig. 2, the sum of every node's battery capacity is lower by MBCR than the one by DSR. Both of avoiding falling out nodes and keeping large number of alive nodes are important for high availability. However excessive protection of low battery nodes increases the total power consumption in entire MANET. It might result in falling out many nodes. Thus routing protocols must take a balance between protecting low battery nodes and lowering the total power consumption. As shown in Fig. 2, MBCR might protect low battery nodes too much. We show that by introducing the participation duration, such excessive protection can be avoided.

\section{The PRoposed PROTOCOL}

\section{A. The Need for the Proposed Protocol}

We propose BPMR (Battery Power Management Routing) protocol that keeps large number of alive nodes by considering the participation duration. MANET protocols must consider the limited battery in each node. It must keep more number of nodes alive and construct a dense network topology. However, battery consumption is not the only characteristic to be achieved. Some nodes might leave from MANET immediately after it has finished its necessary communication. These nodes are not beneficial for entire MANET. In contrast, some nodes keep to participate to MANET for a long duration even if it has no communication request. Such a node has contributed to entire MANET. The nodes in MANET voluntarily relay the other nodes' packets. Thus, if the protocol is not fair among nodes, the incentive to join MANET is low and few nodes join to MANET. It results in a sparse (or disconnected) MANET topology and poor availability. In order to achieve fairness, this paper proposes BPMR that considers participation duration. In addition, considering fairness improves the availability because the load is balanced among nodes, the power consumption is also balanced among nodes, and thus the number of falling out nodes decreases. BPMR modifies the path selection algorithm in routing protocols, thus it can be used with any routing protocols. In this paper, we show modifying DSR as an example.

\section{B. Fairness Problem of Relay Burden}

In DSR, the probability to be selected as a relay node does not depend on the participation duration. Before we show the detail of BPMR, we describe SDP (Simple Duration Protocol) that is the main idea of BPMR. SDP selects the relay nodes by considering the participation duration. The participation duration (PD) in the MANET is a criteria that represents the amount of contribution done as a relay node. Some nodes might not have forwarded so many packets during its participation duration, but the participation increased the potential of successful communication, thus we use not the number of forwarded packets but the participation duration as the criteria. The node of short participation duration can be selected as a relay node more than a longer participation duration node. We propose the evaluation equation of each path in SDP as follows.

$$
\text { PathValue }(P)=\sum_{i=2}^{n-1}\left(1-\frac{1}{\frac{P D\left[x_{i}\right]}{32}+1}\right)
$$

where a path in RREP $P=\left(x_{1}, x_{2}, \ldots, x_{n}\right), x_{1}$ is the source node, $x_{n}$ is the destination node, $x_{i}(2 \leq i \leq n-1)$ are the relay nodes, 
and $P D\left[x_{i}\right]$ is $x_{i}$ 's participation duration $\left(0.0 \leq P D\left[x_{i}\right]\right)$.

$$
\text { SelectPath }=\operatorname{argmin}\{\operatorname{PathValue}(P) \mid P \in A\},
$$

where $A$ is the set of paths obtained by RREPs. A node starts recording the participation duration if one of the following events occurs.

1) The node broadcasts a RREQ packet for its first communication request.

2) The node receives a RREQ packet for the first time.

The coefficient value 32 for PD in (3) is obtained as the best value by a simulation. Though the most suitable coefficient of the term of PD depends on the number of nodes, the frequency of participating and leaving, and so on, it does not change so much from 32 in every situation. (3) and (4) mean that the possibility that short participation duration nodes are selected as a relay node is high and the possibility of long participation duration is low. SDP protocol protects long participation duration nodes from battery exhaust. Thus SDP improves the availability of MANET. We show the effectiveness of the proposed protocol by simulations.

We execute the following simulation to show the characteristic of proposed SDP protocol. 50 nodes are randomly located in $500 \mathrm{~m} \times 500 \mathrm{~m}$ space and random communication requests are generated. The moving speed of each node is randomly selected so that the speed is $4 \mathrm{~km} / \mathrm{h}$ in average. The nodes' maximum battery capacity is $5550 \mathrm{mWh}$ $(1500 \mathrm{mAh}, 3.7 \mathrm{~V})$. Each node's initial battery level is randomly selected between $1 \%$ and $100 \%$. We add the following settings for the node participation and exit. In every minute, one randomly selected node exits from MANET and one new node participates to MANET. There are some long duration nodes in MANET. In order to locate long participation duration nodes, initially selected 25 nodes never exit from MANET.

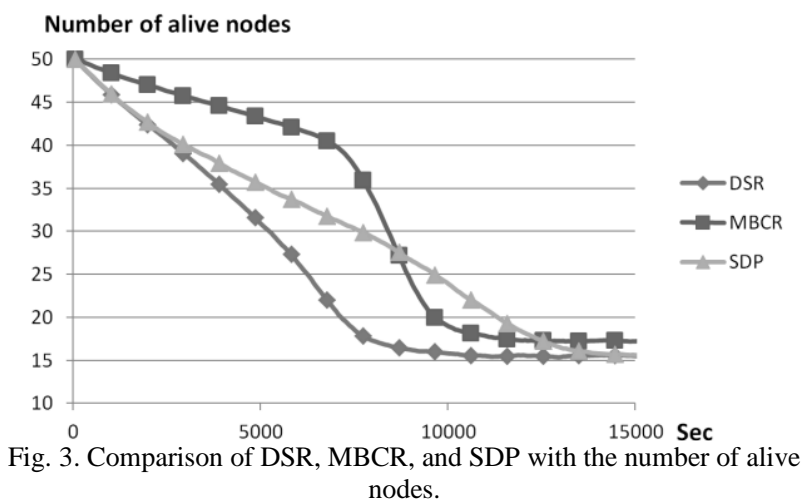

Fig. 3 indicates the number of alive nodes of DSR, MBCR, and SDP. The number of alive nodes is the highest in MBCR before $9000 \mathrm{sec}$. This is because MBCR does not select low battery nodes as a relay node. The number of alive nodes is almost the same in DSR and SDP until $4000 \mathrm{sec}$, because these protocols do not have characteristics of avoiding low battery nodes. However the number of alive nodes is the highest in SDP from about $9000 \mathrm{sec}$. It means that MBCR excessively protects low battery nodes by their neighbor nodes with high battery. Thus, MBCR decreased the sum of remaining battery in all nodes than SDP. It results in the situation that many nodes exhaust their battery after $9000 \mathrm{sec}$.

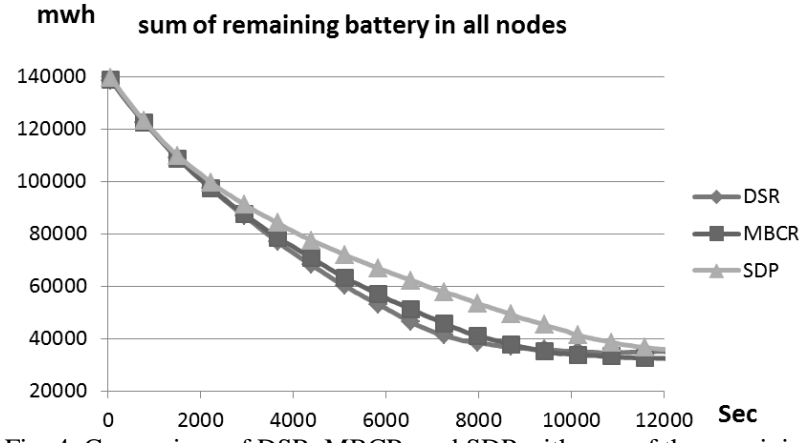

Fig. 4. Comparison of DSR, MBCR, and SDP with sum of the remaining battery capacity of all nodes.

Fig. 4 shows the amount the sum of remaining battery capacity of all nodes by the above simulation. The sum of remaining battery is the highest by SDP among three protocols. Since SDP considers participation duration in the evaluation equation, short participation duration nodes are selected as a relay node more than long duration participation nodes. Such nodes' battery capacities are hard to decrease. Thus, the remaining battery of long participation duration nodes does not decrease within a short period.

However, SDP protocol might select a low battery node as a relay node. Thus some low battery nodes exhaust battery and fall out from MANET and the number of alive nodes decreases. It might result in sparse network topology, few alternative routes for communication, and decreasing the number of reachable nodes. Thus SDP needs to incorporate the characteristics of MBCR that avoids the nodes of low battery level.

\section{Path Selection Algorithm of BPMR}

SDP achieves high remaining battery and thus the number of alive nodes is large when many nodes fall out, but the number of alive nodes is not the best when few nodes fall out. In contrast, MBCR achieves the number of alive nodes large while few nodes fall out. The characteristics of MBCR is very important to maintain alive node ratio. Thus, we incorporate characteristics of MBCR and SDP to BPMR protocol. We modify the path selection algorithm of SDP to consider remaining battery level $(B L)$ as in MBCR.

$$
\operatorname{PathValue}(P)=\sum_{i=2}^{n-1}\left(v \frac{1}{B L\left[x_{i}\right]}+(1-v)\left(1-\frac{1}{\frac{P D\left[x_{i}\right]}{32}+1}\right)\right)
$$

where a path in RREP $P=\left(x_{1}, x_{2}, \ldots, x_{n}\right), x_{1}$ is the source node, $x_{n}$ is the destination node, $x_{i}(2 \leq i \leq n-1)$ are the relay nodes, $P D\left[x_{n}\right]$ is $x_{n}$ 's participation duration $\left(0.0 \leq P D\left[x_{n}\right]\right), v$ is a value representing the weight $(0 \leq v \leq 1)$ of the first term and the second term.

$$
\text { SelectPath }=\operatorname{argmin}\{\operatorname{PathValue}(P) \mid P \in A\},
$$

where $A$ is the set of paths obtained by RREPs.

We show the detail of the path selection algorithm by BPMR. Each node has a value, called Battery Level (BL), which is from 0.0 to 1.0 that indicates its remaining battery ( 0.0 means $0 \%$ and 1.0 means $100 \%$ ). In addition, each node has a value called Participation Duration (PD), which is more than 0.0 that indicates its participation duration (The unit is 
minutes, that is 2.0 means the participation duration is 2 minutes).

1) The same as DSR.

2) A node that receives the RREQ packet checks its whether its destination node is itself. If it is not the destination, it writes its node ID, the remaining battery level (BL), and participation duration (PD) to the RREQ packet and broadcasts the RREQ packet.

3) The same as DSR.

4) The source node selects the route using (5) and (6) to the received RREP packets.

BPMR has the following two characteristics. If the first term (characteristics of MBCR) of (5) is dominant, the power consumption increases. If the second term (characteristics of SDP) of (5) works too much, the number of the falling out nodes increases. The second term effects keeping remaining battery in the entire MANET. Therefore it is necessary to find the most suitable ratio of these two terms. We propose the best value of parameter $v$ by a simulation. The simulation settings is same as the previous one.

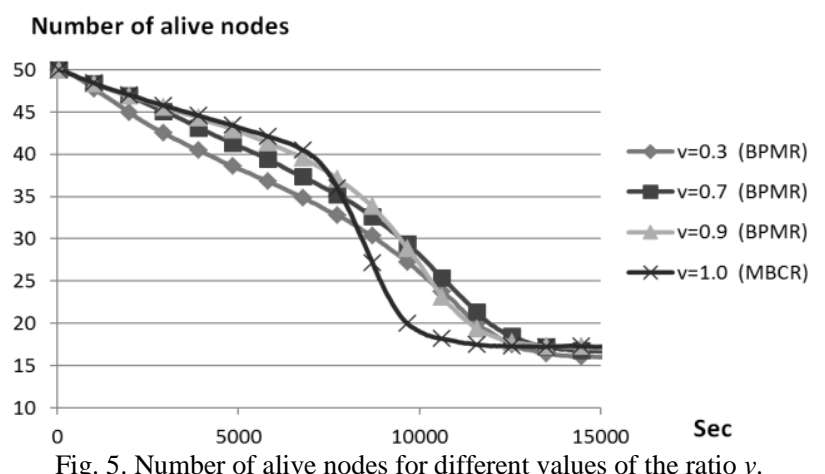

Fig. 5 shows the number of alive nodes for different values of the ratio $v$. In Fig. 5, the number of alive nodes is the largest when $v=0.9$. It means that the weight of the first term (MBCR) is $90 \%$ and the weight of the second term (SDP) is $10 \%$. BPMR selects relay nodes by considering participation duration. Thus early exiting node executes many packet relays. When MANET is operated for a long time, there are many long participation duration nodes. MBCR does not protect such nodes compared with SDP, thus the remaining battery of these nodes decrease. Therefore, the number of alive nodes by MBCR is smaller than the one by BPMR. Thus avoiding these long participation duration nodes results in increasing the number of alive nodes. By BPMR, the nodes have more power resource than by the other protocols from this characteristic.

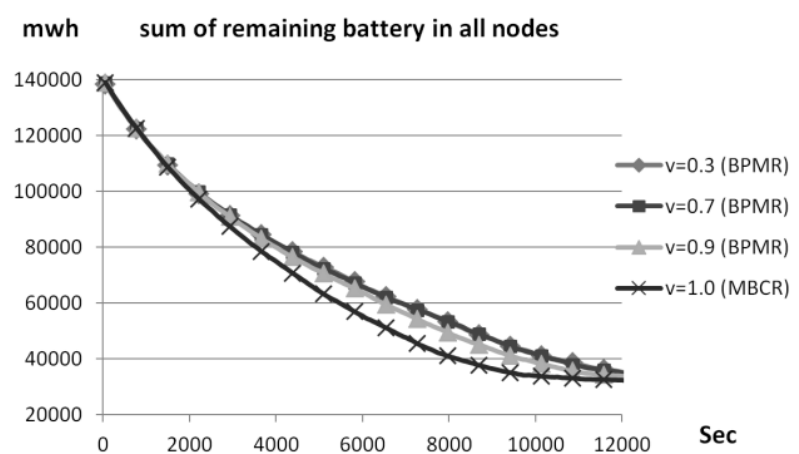

Fig. 6. Sum of remaining battery in all nodes for different values of the ratio
Fig. 6 shows the sum of the remaining battery in all nodes for different values of the ratio $v$ by the above simulation. The sum of the remaining battery in all nodes is the largest when $v=0.3$. However, $v=0.3, v=0.7$, and $v=0.9$ achieve similar values and the difference is very small. Compared BPMRs $(v=0.3,0.7,0.9)$ with MBCR $(v=1.0)$, BPMR achieves better values than MBCR. Thus, we propose the most preferable value of $v$ is 0.9 in BPMR because of the number of alive nodes is the largest when $v=0.9$ in Fig. 5. However, this value can be changed, if a user prefers the number of alive nodes or the sum of remaining battery in all nodes in MANET.

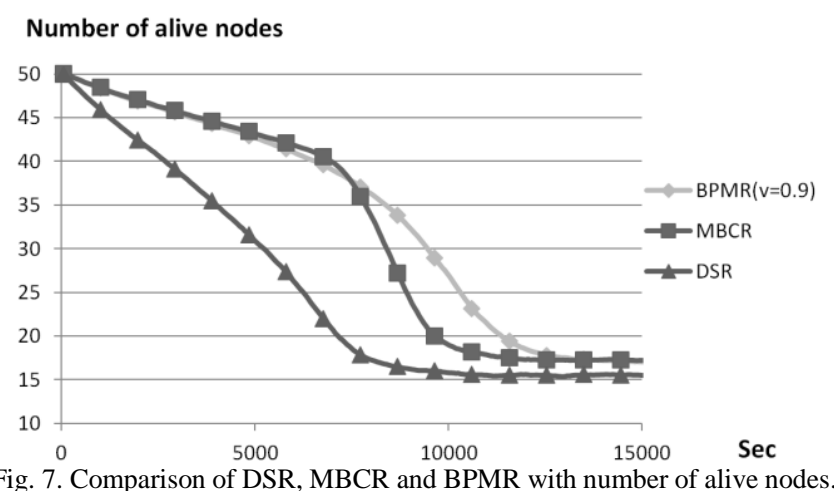

We compare DSR, MBCR and BPMR $(v=0.9)$ by a simulation. Fig. 7 shows the number of nodes for these three protocols. The simulation settings is same as the previous one. The number of alive nodes is almost the same between MBCR and BPMR until $8000 \mathrm{sec}$. From $8000 \mathrm{sec}$ to 12000 sec, the number of alive nodes by BPMR is better than the one by MBCR. Thus, for the number of alive nodes, BPMR is the best algorithm for the entire period.

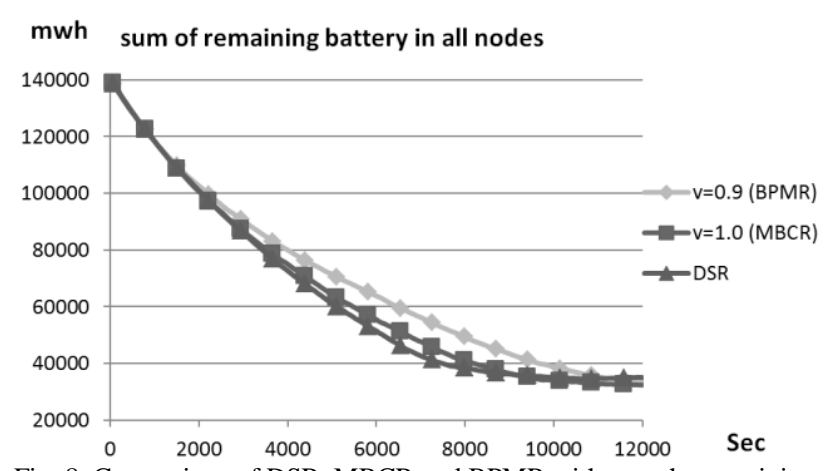

Fig. 8. Comparison of DSR, MBCR and BPMR with sum the remaining battery capacity of all nodes.

Fig. 8 shows the sum of the remaining battery in all nodes for these protocols by the above simulation. In Fig. 8, compared with the other protocols, the remaining battery is the largest in BPMR among the three protocols. This is because BPMR reduces the power consumption of the long duration node with participation duration (PD). BPMR achieves larger remaining battery than the other protocols from $4000 \mathrm{sec}$. In BPMR, the task to relay packets is assigned to short participation duration nodes. The other protocols do not have such a feature. Thus the remaining battery of long participation duration nodes is larger by BPMR than the other protocols and the sum of remaining battery is larger than the other protocols. 


\section{CONCLUSION}

This paper proposed BPMR, a new routing protocol for MANET, in which the route selection is modified from DSR. BPMR uses each node's battery level and participation duration. Long participation duration nodes are not selected as a relay node, thus the fairness among nodes can be achieved. In addition, this characteristics helps increasing the remaining battery. Even if some nodes have little remaining battery, it does not affect the possibility of MANET communication if the nodes exit from MANET within a short period. By selecting short participation duration nodes as a relay node, fairness among nodes can be achieved and the number of alive nodes increases. When a node participates for a long duration, the probability to be selected as a relay node decreases by BPMR. It means that the node is motivated to participate MANET for a long duration. We showed the effectiveness of BPMR by simulations. There is another fairness problem in MANET that is communication amount and frequency within a certain period of time. There are frequent communication request nodes and infrequent communication request nodes. Thus, we think these nodes should not be equally treated in terms of fairness. Achieving this kind of fairness in MANET is our further study.

\section{REFERENCES}

[1] E. M. Royer and C. K. Toh, "A review of current routing protocols for ad-hoc mobile wireless networks," IEEE Personal Communications Magazine, vol. 6, no. 2, pp. 46-55, 1999.

[2] R. Verma, A. Prakash, R. Tripathi, and N. Tyagi, "Throughput enhancement of multi-hop static Ad-hoc networks through concurrent transmission," in Proc. 1st IEEE International Conference on Computational Intelligence on Computational Intelligence, Communication Systems and Networks, 2009, pp. 482-485.
[3] S. Islam, N. Hider, T. Haque, and L. miah, "An extensive comparison among DSDV, DSR and AODV protocols in MANET," International Journal of Computer Applications, vol. 15, no. 2, pp. 22-24, 2011.

[4] C. Yu, B. Lee, and H. Youn, "Energy efficient routing protocols for mobile Ad Hoc networks," Wireless Communication and Mobile Computing, Wireless Com. Mob. Computing, pp. 959-973, 2003.

[5] Q. Dai and J. Wu, "Computation of minimal uniform transmission power in Ad Hoc wireless networks," in Proc. International Conference on Distributed Computing Systems Workshops, 2003, pp. 680-684.

[6] R. Ramanathan and R. Rosales-Hain, "Topology control of multihop wireless networks using transmit power adjustment," in Proc. $9^{\text {th }}$ Annual Joint Conference of the IEEE Computer and Communications Societies, 2000, vol. 2, pp. 404-413.

[7] C. K. Toh, "Maximum battery life routing to support ubiquitous mobile computing in wireless ad hoc networks," IEEE Communication Magazine, vol. 39, no. 6, pp. 138-147, 2001.

[8] B. Johnson and D. Maltz, "The dynamic source routing protocol for mobile Ad Hoc network," RFC 4728, 2003.

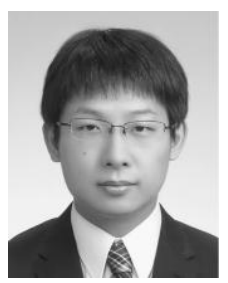

Masaru Yoshimachi was born in 1992. He received his bachelor of informatics degree from Kogakuin University, Japan, in 2015.

Currently, he is a master course student in the Department of Computer Science, Graduate School of Kogakuin University.

Mr. Yoshimachi's research interests include networks, MANET, and routing algorithms.

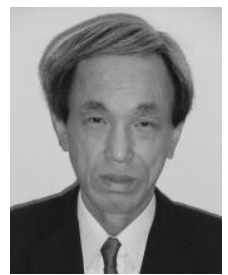

Yoshifumi Manabe was born in 1960. He received his B.E., M.E., and Dr.E. degrees from Osaka University, Osaka, Japan, in 1983, 1985, and 1993, respectively.

From 1985 to 2013, he worked for Nippon Telegraph and Telephone Corporation. From 2001 to 2013 , he was a guest associate professor of Graduate School of Informatics, Kyoto University. Since 2013, he has been a professor of the Faculty of Informatics, Kogakuin University, Tokyo, Japan. His research interests include distributed algorithms, cryptography, game theory, and graph theory. Dr. Manabe is a member of ACM, IEEE, IEICE, IPSJ, and JSIAM. 